\title{
Endothelial cell colonization and angiogenic potential of combined nano- and micro-fibrous scaffolds for bone tissue engineering
}

\author{
Marina I. Santos ${ }^{\text {a,b,c }}$, Kadriye Tuzlakoglu ${ }^{\text {a,b,d }}{ }^{\text {, Sabine Fuchs }}{ }^{c}$, Manuela E. Gomes ${ }^{\text {a,b }}$, Kirsten Peters ${ }^{\text {, }}$, \\ Ronald E. Unger ${ }^{c}$, Erhan Piskin ${ }^{\mathrm{d}}$, Rui L. Reis ${ }^{\mathrm{a}, \mathrm{b}}$, C. James Kirkpatrick ${ }^{\mathrm{c}, *}$ \\ a 3B's Research Group - Biomaterials, Biodegradables and Biomimetics, Department of Polymer Engineering, University of Minho, Campus de Gualtar, 4710-057 Braga, Portugal \\ ${ }^{\mathrm{b}}$ IBB - Institute for Biotechnology and Bioengineering, PT Government Associated Laboratory, Braga, Portugal \\ ${ }^{\mathrm{C}}$ Institute of Pathology, Johannes Gutenberg University Mainz, Langenbeckstrasse 1, Mainz 55101, Germany

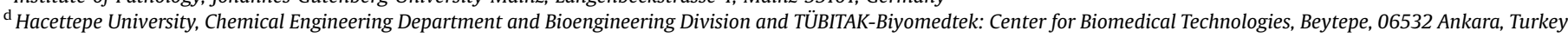 \\ ${ }^{\mathrm{e}}$ Department of Cell Biology, Junior Research Group, Medical Faculty, University of Rostock, Schillingallee 69, 18057 Rostock, Germany
}

\section{A R T I C L E I N F O}

\section{Article history:}

Received 17 July 2008

Accepted 23 July 2008

Available online 15 August 2008

\section{Keywords:}

Starch-based scaffolds

Vascularization

Nano-fibers

Endothelial cells

Bone tissue engineering

\begin{abstract}
A B S T R A C T
Presently the majority of tissue engineering approaches aimed at regenerating bone relies only on postimplantation vascularization. Strategies that include seeding endothelial cells (ECs) on biomaterials and promoting their adhesion, migration and functionality might be a solution for the formation of vascularized bone. Nano/micro-fiber-combined scaffolds have an innovative structure, inspired by extracellular matrix (ECM) that combines a nano-network, aimed to promote cell adhesion, with a micro-fiber mesh that provides the mechanical support. In this work we addressed the influence of this nano-network on growth pattern, morphology, inflammatory expression profile, expression of structural proteins, homotypic interactions and angiogenic potential of human EC cultured on a scaffold made of a blend of starch and poly(caprolactone). The nano-network allowed cells to span between individual micro-fibers and influenced cell morphology. Furthermore, on nano-fibers as well as on micro-fibers ECs maintained the physiological expression pattern of the structural protein vimentin and PECAM-1 between adjacent cells. In addition, ECs growing on the nano/micro-fiber-combined scaffold were sensitive to pro-inflammatory stimulus. Under pro-angiogenic conditions in vitro, the ECM-like nano-network provided the structural and organizational stability for ECs' migration and organization into capillary-like structures. The architecture of nano/micro-fiber-combined scaffolds elicited and guided the 3D distribution of ECs without compromising the structural requirements for bone regeneration.
\end{abstract}

(c) 2008 Elsevier Ltd. All rights reserved.

\section{Introduction}

To become widely used in clinical practice tissue engineering products must overcome a series of major challenges, the vascularization of the biomaterial constructs being one of the major current limitations [1-3]. To date, most approaches in tissue engineering have relied on post-implantation neovascularization from the host, but for large and metabolically demanding organs, which rely on blood vessel ingrowth, this is clearly insufficient to meet the implant's demand for oxygen and nutrients [4-6].

In vascularized tissues/organs such as bone a complex network of blood vessels is more than just simple conduits that provide nutrients and oxygen and simultaneously remove by-products. They also have important metabolic and rheological functions

\footnotetext{
* Corresponding author. Tel.: +49 6131 177301; fax: +49 613117477301.

E-mail address: kirkpatrick@ukmainz.de (C.J. Kirkpatrick).
}

which are organ-specific [7-9]. In bone, the intraosseous circulation allows traffic of minerals between the blood and bone tissue, and transmits the blood cells produced within the bone marrow into the systemic circulation $[9,10]$. New blood vessels are intimately involved in osteogenesis (intramembranous and endochondral) and, furthermore, cytokines and growth factors that regulate intraosseous angiogenesis also regulate bone remodelling [7,9]. In addition, vascularization is also vital for the survival of the implanted cells on the carrier material after implantation [6].

Many approaches have been proposed to increase vascularization in bone such as gene and/or protein delivery of angiogenic growth factors [11,12], provision of a vascularized bone flap [13,14] and ex vivo culturing of scaffolds with ECs alone or in combination with other cell types $[6,15]$. Recently the work of Levenberg et al. on skeletal muscle showed that pre-vascularization of constructs improved in vivo performance of the tissue construct, shedding light into ex vivo use of ECs to accelerate vascularization [16]. Thus, the scaffold design must not only take into consideration the 
structural and mechanical properties of bone but also ECs' adhesion, migration and blood vessel formation and ingrowth. In blood vessels ECs are attached as a monolayer to a basement membrane composed of protein fibers in the nanoscale, such as type IV collagen and laminin fibers, embedded in heparin sulfate proteoglycan hydrogels $[17,18]$. This natural extracellular matrix (ECM) provides structural and organizational stability to ECs and during angiogenesis EC migration is dependent on the adhesion to this matrix [19].

In this present work we evaluate the interaction of ECs with a scaffold made from a blend of starch with poly(caprolactone) (SPCL) with an innovative structure, inspired by the ECM, and combining polymeric micro- and nano-fibers in the same construct. This architecture was designed for bone regeneration to simultaneously provide mechanical support and to mimic the physical structure of ECM. We hypothesized that the presence of a nanonetwork might favour the adhesion of ECs and increase the density of cell colonization between micro-fibers, and might thus accelerate vascularization of the implanted scaffold. Previous work demonstrated favourable activity and differentiation of bone-like cells on this nano/micro-fiber-combined scaffold [20]. In this paper we addressed several important biological questions, such as whether this nano-network favours the growth pattern of ECs on the scaffold, cell morphology, inflammatory gene expression profile, expression of structural proteins and finally the angiogenic potential.

\section{Materials and methods}

\subsection{Scaffolds}

The scaffolds used in this study were based on a blend of starch with poly (caprolactone) (SPCL, 30/70 wt\%). Nano/micro-fiber-combined scaffolds resulted from a two-step methodology. First by a fiber bonding methodology an SPCL fibermesh scaffold composed of micro-fibers $(\varnothing 160 \mu \mathrm{m})$ with $70 \%$ porosity was obtained and second, by electrospinning the scaffold was impregnated with nano-fibers $(\varnothing 400 \mathrm{~nm})$. SPCL fiber-mesh scaffold without the nano-network was used as control. Further details concerning scaffold production have been published elsewhere [20-22]. Samples were cut into discs of $8 \mathrm{~mm}$ diameter and $2 \mathrm{~mm}$ height and sterilized by ethylene oxide. Prior to cell seeding scaffolds were soaked overnight in medium without serum.

\subsection{Cells, culture conditions and scaffold seeding}

Primary cultures of human ECs isolated from umbilical cord (human umbilical vein EC/HUVEC) and from human dermis (human dermal microvascular EC/HDMEC) were used. HUVECs were isolated from umbilical vein by collagenase digestion according to a published method [23]. HDMECs were obtained from enzymatic digestion of juvenile foreskin as previously described [24]. HUVECs were cultured in M199 medium (Sigma-Aldrich, Germany) supplemented with 20\% fetal calf serum (FCS; Gibco, Germany), $100 \mathrm{U} / 100 \mu \mathrm{g} / \mathrm{mL}$ Pen/Strep (Sigma-Aldrich, Germany), 2 mm glutamax I (Life Technologies, Germany), $25 \mu \mathrm{g} / \mathrm{mL}$ sodium heparin (Sigma-Aldrich, Germany) and $25 \mu \mathrm{g} / \mathrm{mL}$ endothelial cell growth supplement (ECGS, BD Biosciences, USA). HDMECs were cultivated in Endothelial Basal Medium MV (PromoCell, Germany) supplemented with 15\% FCS (Invitrogen, Germany) $100 \mathrm{U} / 100 \mu \mathrm{g} / \mathrm{mL}$ Pen/Strep (Sigma-Aldrich, Germany), $2.5 \mathrm{ng} / \mathrm{mL}$ basic fibroblast growth factor (bFGF; Sigma-Aldrich, Germany), $10 \mu \mathrm{g} / \mathrm{mL}$ sodium heparin and $100 \mathrm{U} / 100 \mu \mathrm{g} / \mathrm{mL}$ Pen/Strep. In order to promote better cell adhesion, ECs were seeded into culture flasks previously coated with gelatine. All assays were conducted with cells until passage 4 .

Prior to cell seeding scaffolds were coated with a fibronectin solution $(10 \mu \mathrm{g} / \mathrm{mL}$ PBS, Roche, Germany) for $1 \mathrm{~h}$ at $37^{\circ} \mathrm{C}$. Confluent HUVECs and HDMECs were trypsinized and a suspension of $2 \times 10^{5}$ cells was added to each scaffold. The scaffolds were incubated under standard culture conditions $\left(37^{\circ} \mathrm{C}, 5 \% \mathrm{CO}_{2}\right.$, humidified atmosphere).

\subsection{ECs' imaging}

The viability, phenotype and growth of ECs on nano/micro-fiber-combined scaffolds and on SPCL fiber-mesh scaffolds were analyzed by scanning electron microscopy [25] and by confocal laser scanning microscopy (CLSM) after 3 and 7 days. For viability assessment, the EC-seeded scaffolds were incubated for $10 \mathrm{~min}$ in medium supplemented with $0.1 \mu \mathrm{m}$ calcein-AM. Viable cells convert the non-fluorescence and membrane permeable calcein-AM due to the presence of active intracellular esterases into the green fluorescent and impermeable calcein. Viable cells are identifiable by the green fluorescent cytoplasm when viewed with CLSM (Leica TCSN NT). For SEM analysis the samples were fixed for 30 min with $2.5 \%$ glutaraldehyde in $0.1 \mathrm{~m}$ sodium cacodylate buffer, dehydrated in increasing concentrations of acetone, dried with hexamethyldisilazane and sputter coated with gold prior to SEM observation (Leica Cambridge S360).

\subsection{Gene analysis of pro-inflammatory genes}

The gene analysis of two pro-inflammatory cell adhesion molecules E-selectin and intercellular adhesion molecule (ICAM-1) was carried out by Real-time PCR. The mRNA expression of cell adhesion molecules as well as the housekeeping gene GAPDH was analyzed in HUVECs growing for 7 days on SPCL fiber-mesh scaffold and on nano/micro-fiber-combined scaffold. As control HUVECs were grown on cellculture plastic. Furthermore, as positive control, gene expression was analyzed when the samples were cultured in the presence of $1.0 \mu \mathrm{g} / \mathrm{mL}$ of lipopolysaccharide (LPS) for $4 \mathrm{~h}$ (Sigma-Aldrich, Germany). Total mRNA from HUVEC cells was extracted using the RNeasy Mini Kit (Qiagen, Germany) according to the manufacturer's protocol. Afterwards, total RNA $(0.5 \mu \mathrm{g})$ was reverse transcribed using Omniscript RT Kit (Qiagen, Germany). Gene amplification was performed using Applied Biosystems 7300 Real-Time PCR System (Applera Deutschland GmbH, Germany). The number of cycles and annealing temperature were selected according to the manufacturer's instructions. Real-time PCR was performed with $2.5 \mathrm{ng}$ cDNA and $12.5 \mu \mathrm{L}$ of $2 \times$ master mix, primers $(0.25 \mu \mathrm{L}$ forward and $0.25 \mu \mathrm{L}$ reverse primer) in a final volume of $25 \mu \mathrm{L}$. The following gene-specific primer sets were used: (1) E-selectin, sense $5^{\prime}$ CCCGTGTTTGGCACTGTGT-3' ${ }^{\prime}$, antisense $5^{\prime}$-GCCATTGAGCGTCCATCCT- $3^{\prime}$; (2) ICAM-1, sense 5'-CGGCTGACGTGTGCAGTAAT-3', antisense 5'-CACCTCGGTCCCTTCTGAGA-3'; (3) GAPDH, sense 5'-ATGGGGAAGGTGAAGGTCG-3', antisense 5'-TAAAAGCAGCCCTGGTGACC $-3^{\prime}$. Gene expression was normalized to the expression of the housekeeping gene GAPDH. Relative quantification of gene expression was calculated in stimulated samples ( + LPS) compared to samples cultured in the absence of pro-inflammatory stimulus (-LPS).

\subsection{Immunocytochemistry}

The expression pattern of the structural protein vimentin and of the platelet endothelial cell adhesion molecule (PECAM-1, CD31) was examined by immunocytochemistry. After 7 days in culture, EC-confluent SPCL scaffolds were fixed with a solution of $2 \%$ paraformaldehyde for $30 \mathrm{~min}$ at room temperature (RT). Samples were rinsed in PBS and then treated with PBS-buffered 0.1\% Triton X-100 for 5 min at RT to permeabilize the cell membranes for the antibody reactions. The samples were incubated for $45 \mathrm{~min}$ at RT with the primary antibodies: mouse anti-human PECAM1 (1:50, Dako, Denmark) or mouse anti-human vimentin (1:200, Sigma-Aldrich, Germany). Following PBS washing, a second incubation was performed for $45 \mathrm{~min}$ at RT with the secondary antibody anti-mouse Alexa Fluor 488 (Invitrogen, Germany). The nuclei were counterstained with $1 \mu \mathrm{g} / \mathrm{mL}$ Hoechst in PBS for $5 \mathrm{~min}$. SPCL fiber meshes were then washed with PBS, mounted with Gel/Mount (Natutec, Germany) and visualized by CLSM.

\subsection{Induction of angiogenesis in vitro}

The angiogenic potential of HDMEC growing on SPCL fiber-mesh scaffolds was assessed by observing the cell migration from the scaffold into a collagen type I gel that mimics the in vivo microenvironment. When HDMECs reached confluence on the scaffolds the scaffolds were transferred to a Petri dish and covered with a $1.5 \mathrm{mg} /$ $\mathrm{mL}$ solution of collagen type I in M199 medium containing $2 \%$ sodium bicarbonate, $0.05 \mathrm{~m} \mathrm{NaOH}$ and 200 nм HEPES. As soon as the solution solidified into a gel, culture medium supplemented with angiogenic growth factors $50 \mathrm{ng} / \mathrm{mL}$ vascular endothelial growth factor (VEGF; Biomol, Germany) and $10 \mathrm{ng} / \mathrm{mL}$ bFGF was added. After an additional 7 days in culture, materials were examined for the migration of ECs and organization into capillary-like structures after calcein-AM live-staining and visualization by CLSM. All the above-referred reagents were from Sigma-Aldrich, Germany.

In order to have a better perception of the spatial distribution of capillary-like structures and micro-fibers the confocal images were post-processed using the image processing software ITK-SNAP [26]. Individual confocal image stacks from nano/micro-fiber-combined scaffold composed of 99 sections were examined. Capillary-like structures were identified and labelled in green and red, respectively, using the manual segmentation tool and the segmented elements were processed into a final 3D image.

Aimed at the evaluation of EC ultrastructure, transmission electron microscopy (TEM) of collagen gel ultrathin sections was performed. Scaffolds plus collagen gel were fixed in $2.5 \%$ glutaraldehyde in cacodylate buffer, post-fixed in $1 \%$ osmium tetroxide for $2 \mathrm{~h}$ and dehydrated in increasing ethanol concentration. Samples were embedded in agar resin 100 (PLANO, Germany) with ethanol as solvent for transition state and subjected to polymerization at $60^{\circ} \mathrm{C}$ for $48 \mathrm{~h}$. Ultrathin sections were cut, placed onto copper grids and examined by transmission electron microscope (Philips EM 410). 


\section{Results}

3.1. Growth, viability and phenotype of ECs on starch-based scaffolds

ECs of microvascular origin, HDMECs as well as the macrovascular HUVECs grew on both fibronectin-coated nano/microfiber-combined scaffolds and on SPCL micro-mesh scaffolds.
Growth was observed on both micro- and nano-fibers (Fig. 1). The requirement of a pre-coating with fibronectin or other ECM molecule for EC adhesion to several substrates has been widely reported [27-29]. On nano/micro-fiber-combined scaffolds, after 3 days of culture HDMEC spanned between adjacent micro-fibers using the nanobridges formed by the nano-fibers, thus yielding a high density of adherent ECs (Fig. 1A). After 7 days, nearly complete growth on the surface areas of the scaffold was observed
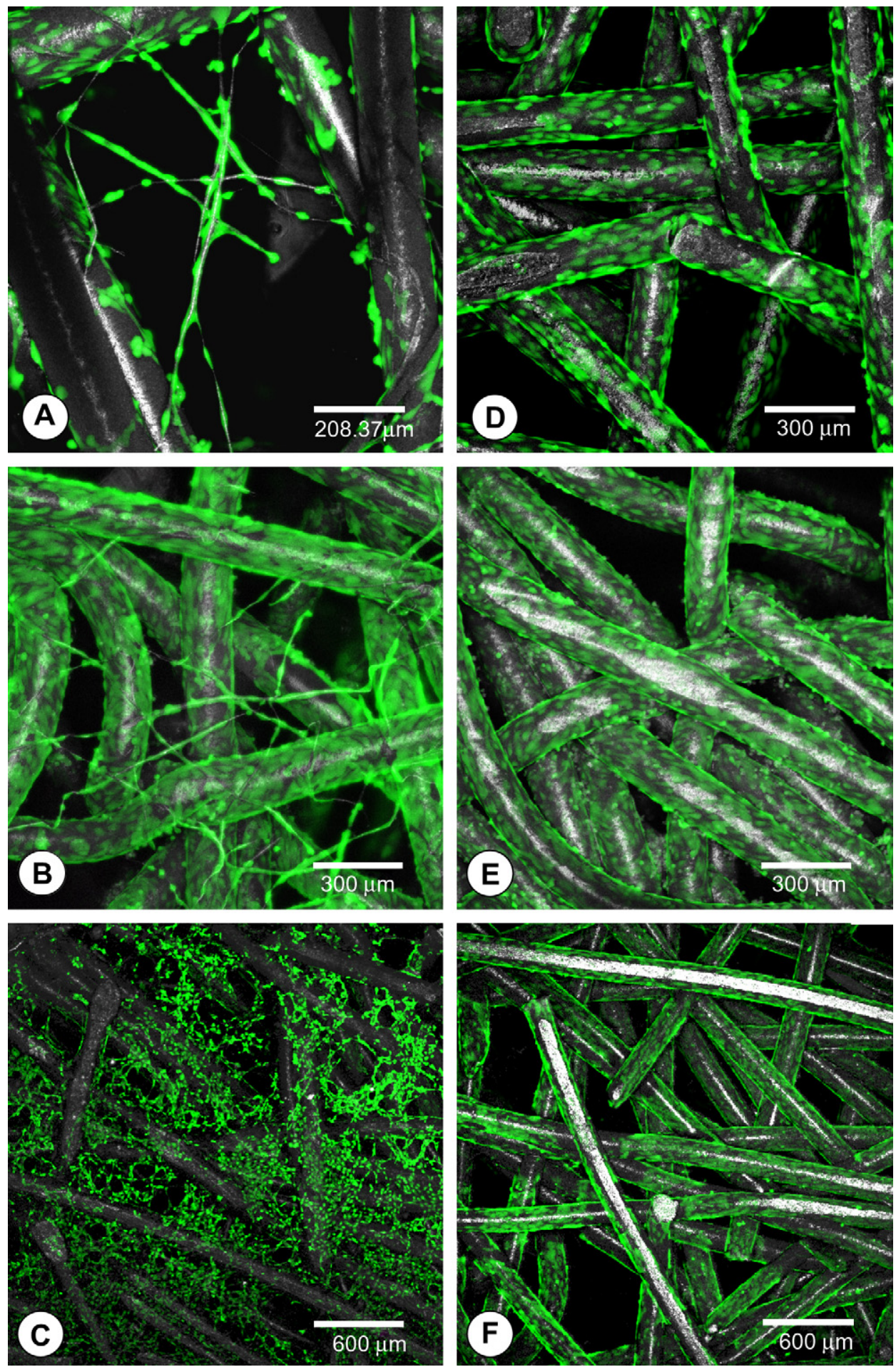

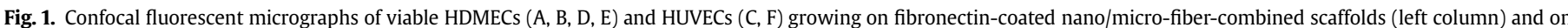

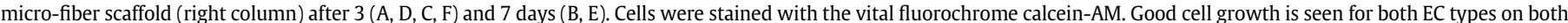
nano- and micro-fibers. The values of the scale bars are: (A) $208 \mu \mathrm{m}$, (B, D, E) $300 \mu \mathrm{m},(C, F) 600 \mu \mathrm{m}$. 
(Fig. 1B). HDMECs were found on both micro- and nano-fibers and remained viable as proven by the ability to convert calcein-AM into a green fluorescent compound. On the other hand, on the scaffold without the nano-network, cells were detected on the micro-fibers after 3 days as well as after 7 days, but no cells were seen to span between the fibers (Fig. 1D and E).

Cell adhesion studies were also performed with primary cultures of macrovascular ECs, HUVECs. HUVECs seeded onto nano/ micro-fiber-combined scaffolds rapidly covered the entire surface of the nano-network without significantly impairing the scaffold porosity (day 3, Fig. 1C). On the other hand, viable HUVECs adhered to the individual fibers on SPCL micro-fiber-mesh scaffold (Fig. 1F).

Phenotype of HUVECs was assessed by SEM after 3 days of culture. ECs adhered to the randomly electrospun nano-network as well as to micro-fibers and cells used the nano-fibers to bridge empty spaces in the micro-fiber mesh (Fig. 2A). In contrast, the SPCL micro-fiber-mesh scaffold did not induce cell spanning across the construct (Fig. 2C). Morphologically, ECs on micro-fibers exhibited the typical flattened phenotype of ECs (Fig. 2C) whereas the nano-network induced a different cytoskeletal arrangement reflected in the stretched shape and numerous cellular protrusions (Fig. 2A). Besides improving the interconnectivity in the scaffold, nano/micro-fiber-combined scaffolds also provided a unique physical support that allows the growth of ECs into circular arrangements that resemble the morphology of capillary-like structures (Fig. 2B).

\subsection{Expression of genes involved in the inflammatory response}

In blood vessels the endothelium functions as a dynamic and actively transporting barrier, which under special conditions, such as inflammation, mediates leukocyte recruitment by the expression of different cell adhesion molecules like ICAM-1 (intercellular adhesion molecule-1) and selectins. Utilizing Real-time PCR the expression of two cell adhesion molecules E-selectin and ICAM-1 on HUVECs growing on nano/micro-fiber-combined scaffolds was analyzed and compared with the expression on control scaffolds (SPCL micro-fiber-mesh scaffold without nano-fibers). HUVECs grown on tissue culture plastic served as control (Fig. 3). ECs growing on the three substrates reacted in a similar way when exposed to the pro-inflammatory stimulus LPS, increasing the levels of mRNA that code for ICAM-1 and E-selectin. As a common pattern it was observed that the up-regulation of E-selectin was higher than ICAM-1 in response to LPS. This lower level of upregulation of ICAM-1 relatively to E-selectin is probably due to the constitutive expression of this cell adhesion molecule on ECs and consequently to a minor difference between the basal and stimulated state. With respect to the combined scaffold, the presence in the same construct of micro- and nanometric fiber size did not affect the ability of ECs to properly respond to pro-inflammatory stimuli through the up-regulation of these genes related to the capture of circulating leukocyte, this representing an essential stage in the physiological inflammatory reaction.

\subsection{Expression of the structural protein vimentin and the cell-cell adhesion molecule PECAM-1}

Cell structure and the interactions with neighbouring cells are important aspects to take into consideration in studying cell functionality. Thus, the expression of vimentin, an intermediate filament protein present in mesenchymal cells and of PECAM-1 (CD 31 ), a cell adhesion molecule present predominantly at the intercellular junctions was assessed by immunocytochemical staining. Fig. 4A shows that on nano-fibers, endothelial vimentin filaments are more stretched, but no disruption of this structural protein was
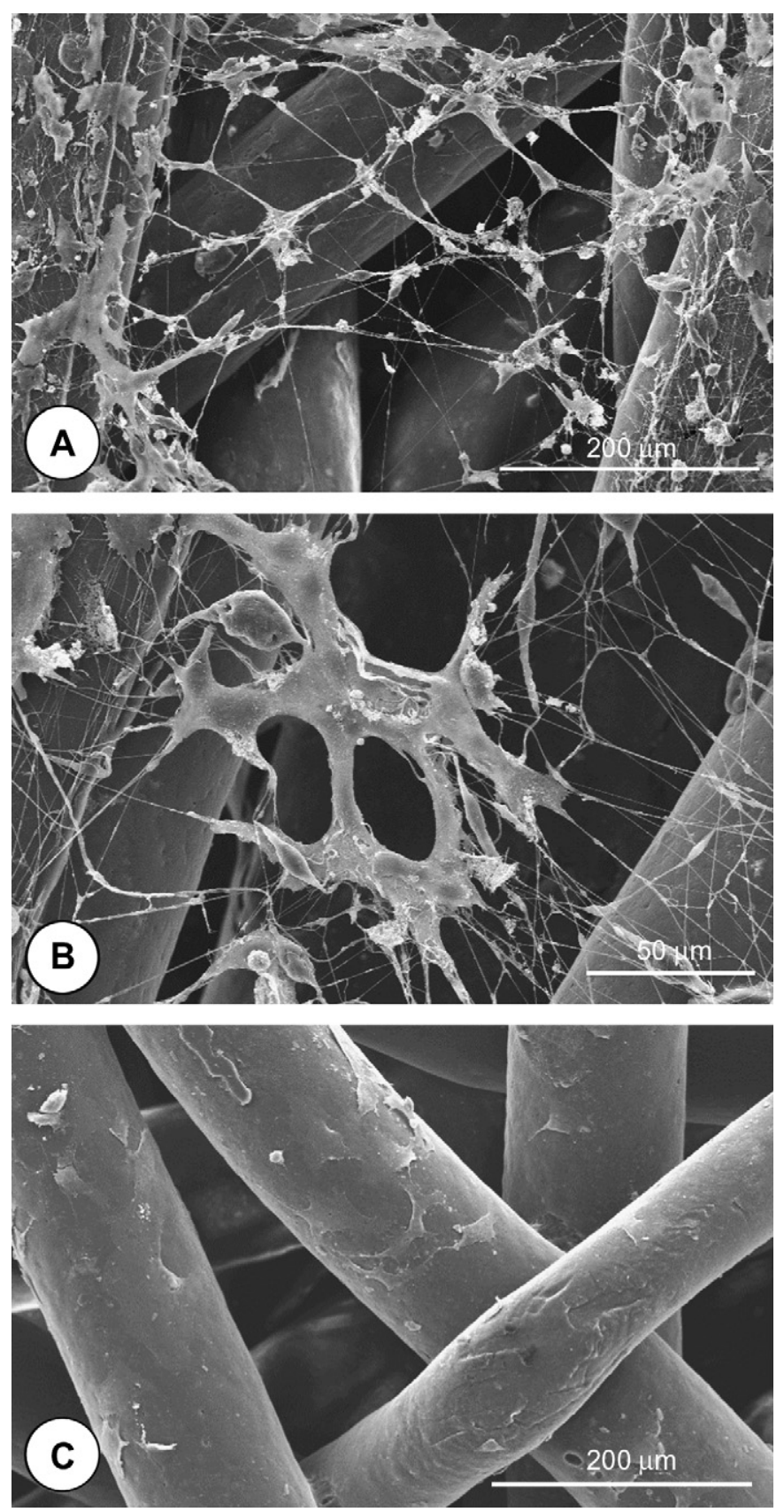

Fig. 2. SEM micrographs of HUVEC cells on fibronectin-coated SPCL scaffolds: (A, B) nano/micro-fiber-combined scaffold and (C) micro-fiber scaffold after 3 days of culture. Note the ability of the EC to use the nanofibers to span across the micro-fiber structure.

observed. Elongated, vimentin-stained cells populated the entire scaffold and grew along both nano- and micro-fibers.

A typical PECAM-1 expression pattern (a peripheral ring surrounding cells at cell-cell interfaces) was observed on both nano-micro-fiber-combined scaffolds and on control scaffolds (Fig. 4B and C). ECs on nano-fibers as well as on micro-fibers continued to express this major cell adhesion molecule. This indicates that despite the differences in the dimensions of the underlying substratum ECs can still establish contact with adjacent cells.

\subsection{Angiogenic potential of ECs on starch-based scaffolds}

In a more complex in vitro assay, the ability of ECs in contact to the scaffolds to invade into and to form capillary-like structures 


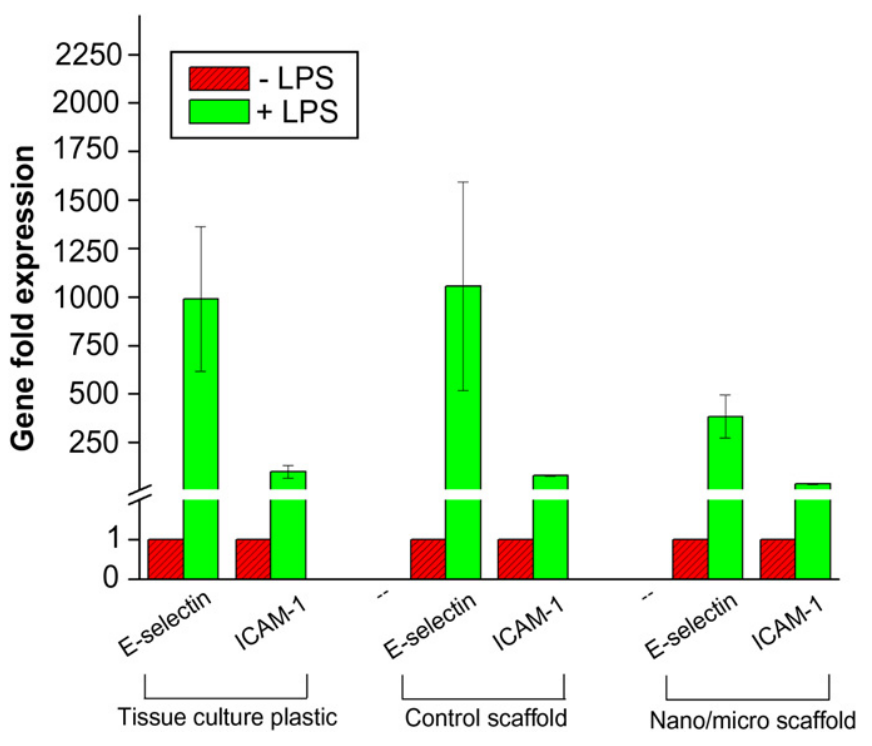

Fig. 3. Relative quantification of E-selectin and ICAM-1 mRNA in HUVECs grown on nano/micro-fiber scaffolds and on micro-fiber scaffolds in the presence of LPS (+LPS) compared with the growth in the absence of pro-inflammatory stimulus (-LPS). As a control the expression of these inflammatory genes was assessed on HUVECs growing on cell-culture plastic.

within a 3D-gel of collagen in response to angiogenic factors was assayed. To visualize the cells within the 3D-matrix a calcein-AM staining was necessary. This staining revealed that ECs were able to migrate from the scaffold and invade into the collagen gel on both nano/micro-fiber-combined scaffolds as well as in control scaffolds, after 7 days of culture (Fig. 5). However, a different behavior between the two scaffolds was observed. On the micro/nano-fibercombined scaffold, ECs formed more capillary structures with branching (Fig. 5A). In contrast, on the scaffolds without the nanonetwork, ECs formed fewer capillary-like structures with less branching (Fig. 5C).

The 3D reconstitution of the segmented micro-fibers and capillary-like structures from the confocal stack images of nano/ micro-fiber-combined scaffold (Fig. 5A is the projection) provided further information. It was shown that the tubular structures formed by ECs were present at different depths, and that there was a spatial separation between the capillary-like structures and the micro-fibers along the $Z$ axis (Fig. 5D). Thus, this further reinforces the ability of ECs to migrate out of the scaffold into the collagen matrix and to organize into capillary-like structures.

The ultrastructure of ECs assessed by TEM revealed the existence of adherent junctions between ECs, denoting the intimate contact between angiogenesis-stimulated cells (Fig. 6A). At a higher magnification numerous vesicles were visible, indicative of the highly active state of the ECs (Fig. 6B).

\section{Discussion}

Tissue engineering scaffolds should function as temporary ECMs and until repair or regeneration occurs they should aim to mimic native ECM both architecturally and functionally [30]. In physiological tissue re-organization (e.g. during wound healing) the bidirectional flow of information exchanged between cells and ECM steers important cell functions such as adhesion, differentiation and migration [31]. Thus, it can be suggested that the more a scaffold can resemble ECM, the more successful the scaffold can be. To date, electrospinning has been one of the main processing techniques used in the fabrication of structures in the nanometer range. This fiber spinning technique produces polymer fibers with diameters down to a few nanometers and nano-fibers obtained by electrospinning have been proposed for engineering many different tissues [32]. However, electrospun scaffolds retain several problems such as three-dimensional cell growth restricted to a depth of $100 \mu \mathrm{m}$, lack of control of pore diameter and distribution, as well as low stiffness [33].

Tuzlakoglu et al. proposed nano/micro-fiber-combined scaffolds, a matrix that combines two structures: (i) a nano-network produced by electrospinning, that mimics ECM and aims to increase cell adhesion and motility; with (ii) a micro-fiber-mesh produced by fiber bonding aimed to give the mechanical support required during repair [20]. This latter structure, an SPCL fiber-mesh scaffold, was used in this work as a control and it was previously described by our group as a promising biomaterial for bone regeneration $[21,34,35]$. Studies with bone marrow cells cultured under dynamic conditions on SPCL fiber-mesh scaffolds showed that cells differentiated into osteoblasts deposited a mineralized matrix and produced several bone growth factors [21,34,35]. Furthermore, previous work with ECs revealed that they maintained their genotypic and phenotypic patterns when growing on SPCL fibermesh scaffolds [36].

Based on the previous studies that have proven the suitability of nano/micro-fiber-combined scaffold for osteoblast differentiation and activity [20], the present work deals with the influence this ECM-like architecture has on EC growth pattern, homo- and heterotypic interactions and on angiogenic potential.

Cell adhesion studies with HDMECs and HUVECs revealed that both cell types adhere and remain viable on fibers in the nano- as well as in the micrometer range. In fact, in the nano/micro-fibercombined scaffold the existence of a structure that resembles the physical structure of ECM furnishes the physical points required for ECs to span between the bulk structure of the scaffold without compromising the porosity and interconnectivity of the structure. Moreover, these nanometer dimensions are reflected in individual cell phenotype and overall cellular rearrangement. On the microfibers the cells exhibit the same flattened morphology characteristic of their location inside larger blood vessels. By contrast, on the nano-network ECs present an extremely stretched shape reminiscent of the angiogenic phenotype, with multiple cellular protrusions anchoring them to several nano-fibers. This stretched phenotype might be beneficial as it was reported that ECs spreading or elongating show increased sensitivity to specific growth factors such as bFGF [37]. Furthermore, the nano-fibers allow a more comprehensive arrangement of ECs positioned within the scaffolds when compared with the scaffolds without nanofibers. Thus, ECs could be easier available for blood vessel formation after implantation of constructs. Of special interest is the capability of the adherent cells to use the physical support provided by the nano-network to adhere and spread into circular arrangements that morphologically resemble capillary-like structures.

Bone tissue-engineered constructs should not only induce good phenotypic properties in ECs such as spreading morphology, cell viability, and cell attachment but also encourage ECs' cell functions, which can be assessed through the expression of cell-cell adhesion molecules involved in heterotypic (cell adhesion molecules such as ICAM-1) and homotypic (PECAM-1) interactions as well as in migration studies. Concerning the heterotypic interactions, ECs play a key role during the inflammatory response through the sequential expression of cell adhesion molecules [38]. These molecules recognize specific ligands on circulating leukocytes and help them to transmigrate across the endothelium towards the proinflammatory stimuli, thus enabling inflammation. In a scaffold for tissue regeneration it is necessary that in the presence of a proinflammatory stimulus, such as cytokines like TNF- $\alpha$ or bacterial compounds like endotoxins, ECs possess the potential to participate through the expression of adhesion molecules for leukocytes. That 

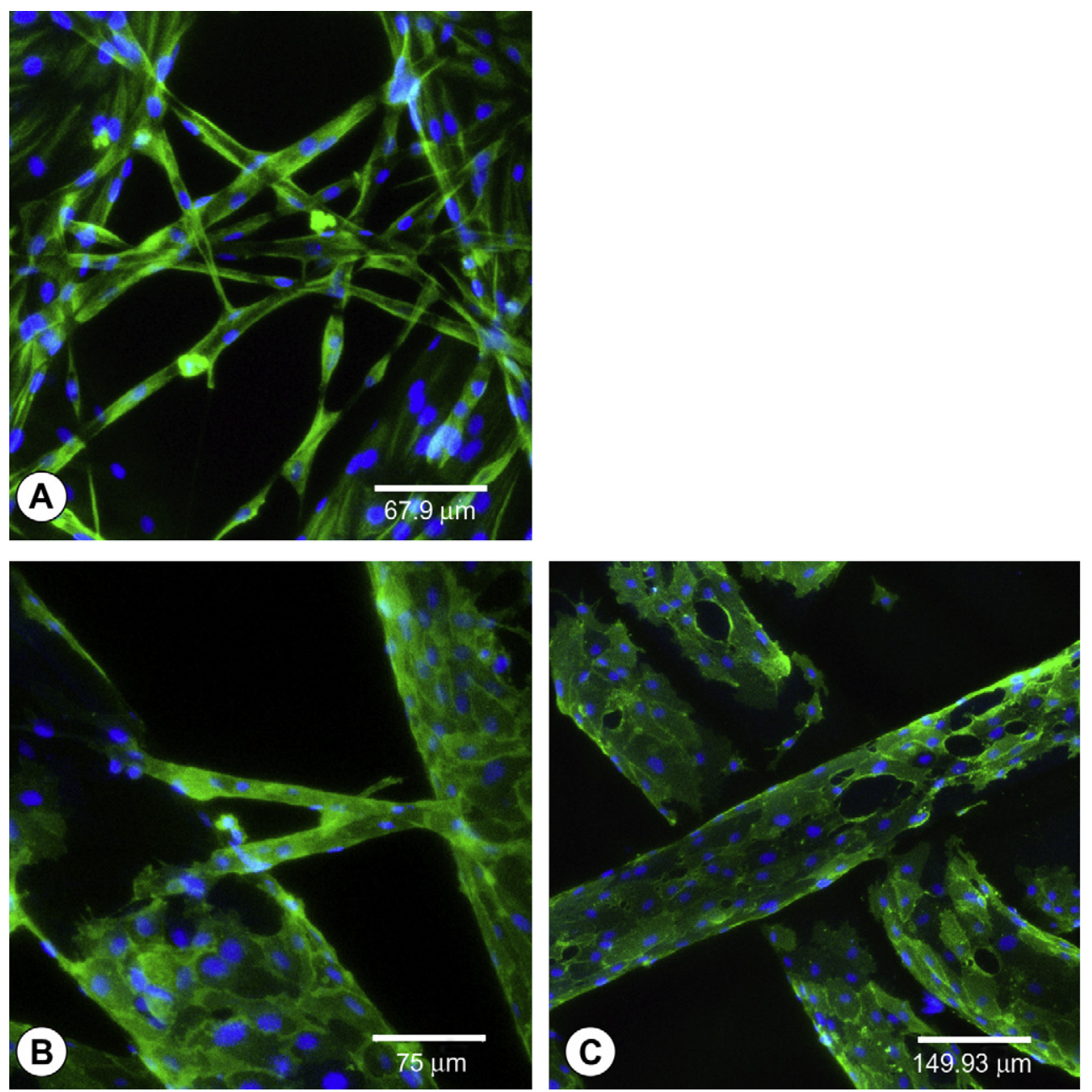

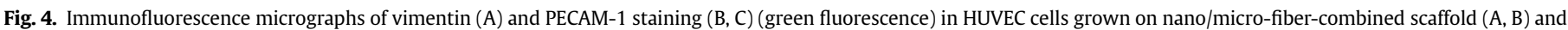
on micro-fiber scaffold (C). Nuclei were counterstained with Hoechst (blue fluorescence). The values of the scale bars are: (A) $68 \mu \mathrm{m}$, (B) $75 \mu \mathrm{m}$, (C) $150 \mu \mathrm{m}$.

means that scaffolds must allow ECs to participate adequately during an inflammatory response but in the absence of an inflammatory stimulus should not induce up-regulation of cell adhesion molecules. This last situation could lead to a continuously inflammatory activated endothelium and consequently to a massive recruitment of leukocytes and increased vascular permeability. To this end, the possible interference of the nano/ micro-fiber-combined scaffold on gene expression of two cell adhesion molecules characteristic for pro-inflammatory activation of ECs, E-selectin and ICAM-1, was analyzed by Real-time PCR. The results showed the ability of ECs on nano/micro-fiber-combined scaffold to up-regulate the expression of ICAM-1 and E-selectin in response to the pro-inflammatory stimulus LPS, following the same pattern observed for the scaffold- and tissue culture plastic controls. This not only suggests the capacity of cells growing on nano/micro-combined scaffolds and on control scaffolds to participate in the inflammatory response through the expression of pro-inflammatory genes, but also indicates that under normal conditions (absence of LPS) the growth of ECs on these scaffolds does not appear to affect the expression of these genes.

The interendothelial interactions of endothelial cells on the scaffolds were evaluated by PECAM- 1 distribution pattern. When studying the interaction of tissue-engineered scaffolds with ECs it is important to assess PECAM-1 distribution pattern, not only because it is a major endothelial marker but also due to the key role this protein plays in endothelial barrier integrity and in leukocyte transmigration during the inflammatory response [39]. On SPCL fibers in the nano- and micro-range, PECAM-1 is present predominantly in the contact sites at cell-cell borders. These cell-cell contact sites are a positive indication of the adequate interendothelial contacts established between adjacent cells when growing and are typical of a quiescent (non-stimulated) endothelium. This is particularly relevant for the nano-network as the effects of electrospun nano-fibers on the phenotypic behavior of a variety of cell types have been previously reported [30]. In order to examine endothelial cell structure we performed immunostaining for vimentin on cells growing on fibers. Vimentin is an intermediate filament protein responsible for maintaining cell shape, integrity of the cytoplasm, and stabilizing cytoskeletal interactions [40]. Vimentin filaments in the cells growing on the nano-network were more stretched than those on micro-fibers but exhibited no apparent disruption of cell structural integrity.

A successful scaffold for bone regeneration must not only promote osteogenesis but also promote the development of a vascular network. Post-natal vascularization occurs mainly by angiogenesis, which is a multi-stage process characterized by an orderly sequence of events including matrix degradation, EC migration, proliferation and formation of new basement membrane components [41]. Migration is driven chemotactically via gradients of cytokines or other agonists but it is the fibrillar structure of ECM in the nanometer range of dimensions that provides the physical cues to proliferating and migrating ECs to organize and form new 

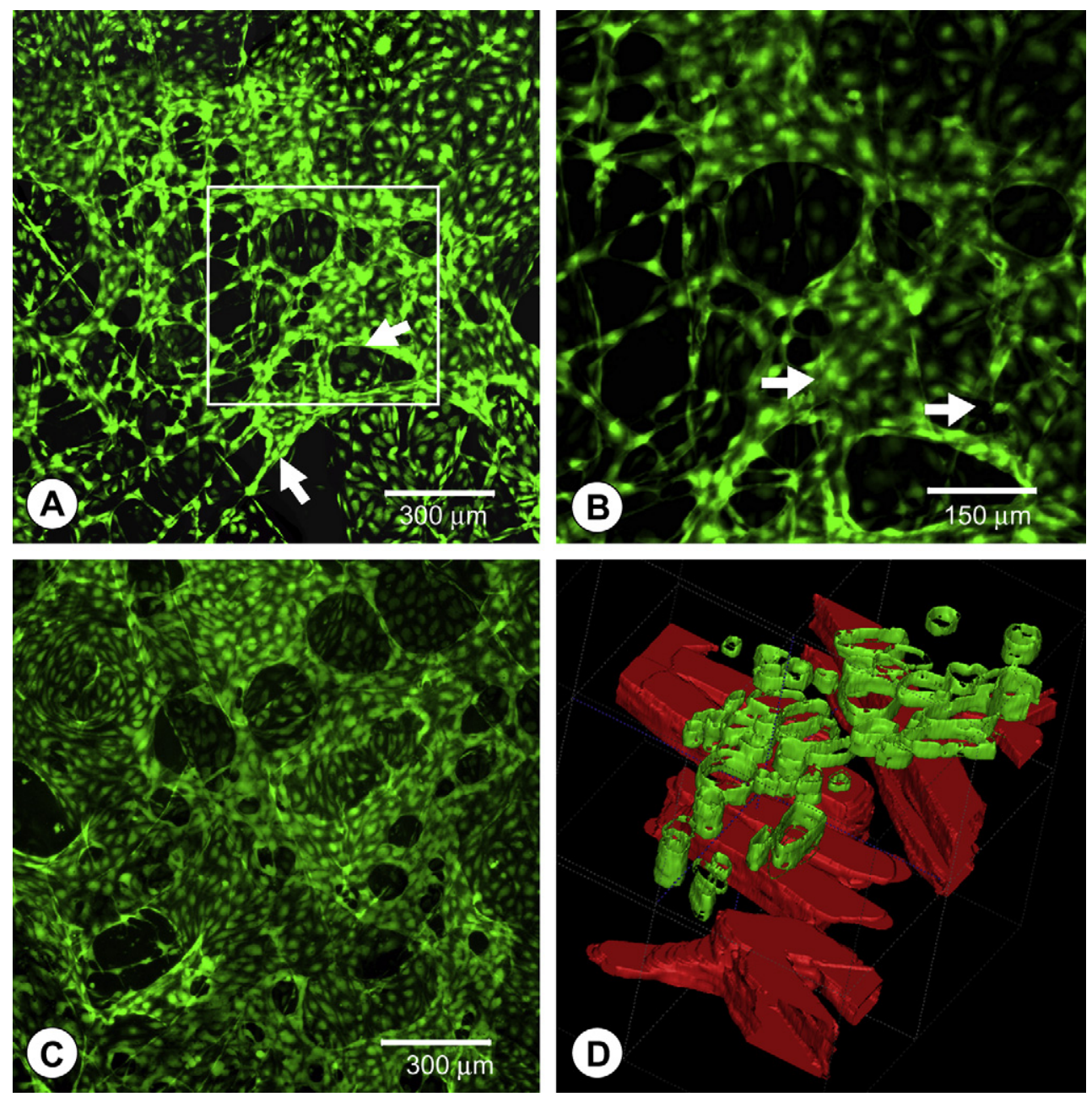

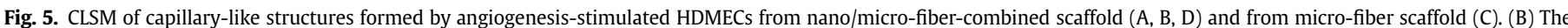

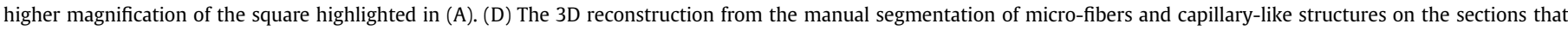

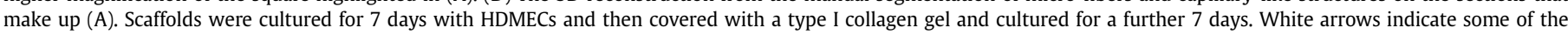
capillary-like structures. The values of the scale bars are: (A) $300 \mu \mathrm{m}$, (B) $150 \mu \mathrm{m}$, (C) $300 \mu \mathrm{m}$.

3D capillary networks [19]. The angiogenic potential of ECs on the scaffold with ECM-like structure under a pro-angiogenic environment was examined in order to determine if nano-structures affected this process. ECs migrated from both scaffolds into the collagen gel, but there appeared to be an elevation in migration and $3 \mathrm{D}$ organization into capillary-like structures in the nano/micro- combined scaffold. This achievement in the later scaffold could be due to the increased surface area, the ECM-like structure and to the elongated cell morphology. Folkman et al. reported that confluent ECs are refractory to growth factors, whereas stretched or elongated ECs have increased sensitivity to growth factors, such as bFGF [37]. These findings might indicate that stretched cells on nano-
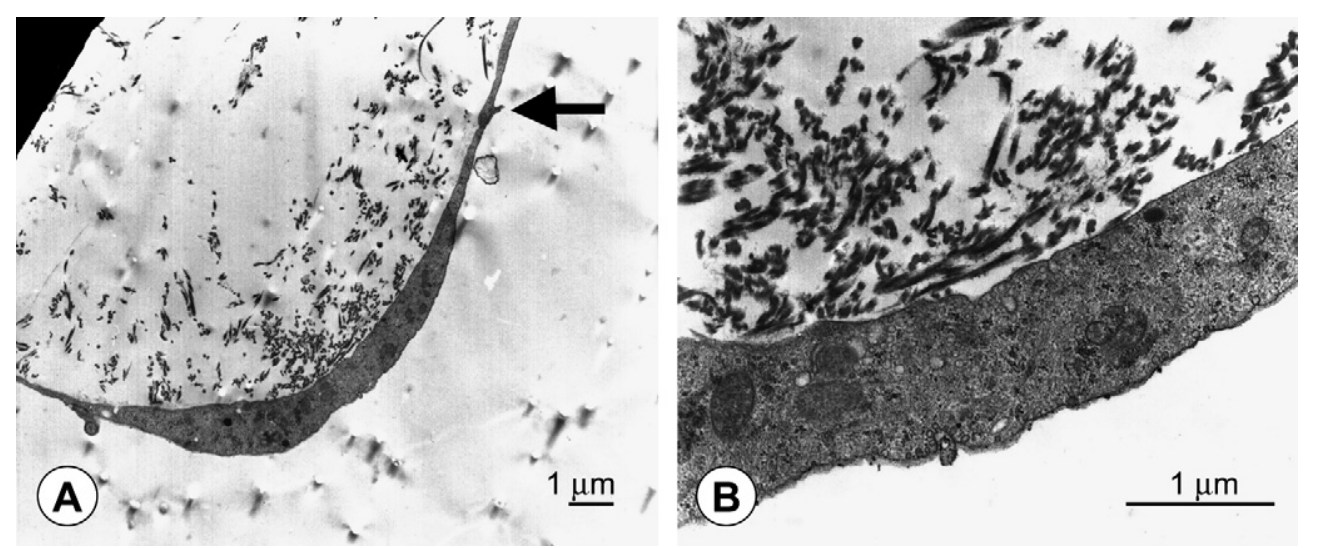

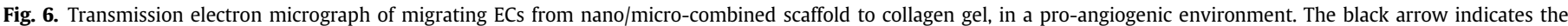
intimate contact between two ECs. 
fibers are more responsive to angiogenic growth factors thus organizing more easily into capillary-like structures, than confluent and less sensitive cells on micro-fibers. Whether this is the case, needs to be investigated in further studies which quantitate the complex three-dimensional reaction.

\section{Conclusion}

The incorporation of structures that physically mimic the ECM, i.e., nano-networks on micro-fiber meshes, not only increased the adhesion surface area and interconnectivity in the constructs but also provided structural and organizational stability for ECs. Using nano-fibers as bridges both human micro- and macrovascular ECs spanned between micro-fibers exhibited a more stretched phenotype when compared with the scaffold without nano-fibers. Furthermore, once the nano-fibers allowed a comprehensive arrangement of ECs in the in vitro constructs, ECs could be easier available for blood vessel formation after implantation of constructs. Furthermore, ECs on nano- as well on micro-fibers maintained their structural integrity (vimentin) and their intercellular contacts (PECAM-1). Moreover, ECs growing on nano/micro-combined scaffolds exhibited a marked angiogenic potential as shown by their ability to form extensive networks of capillary-like structures.

\section{Acknowledgements}

M.I. Santos would like to acknowledge the Portuguese Foundation for Science and Technology (FCT) for her PhD scholarship (SFRH/BD/13428/2003). This work was partially supported by FCT through funds from POCTI and/or FEDER programs and by the European Union funded STREP Project HIPPOCRATES (NMP3-CT2003-505758). This work was carried out under the scope of the European NoE EXPERTISSUES (NMP3-CT-2004-500283).

The authors would also like to acknowledge the excellent technical assistance of A. Sartoris, B. Pavic, S. Barth, M. Muller and K. Molter. We greatly appreciate the help provided by Hui Zhang with the image processing software ITK-SNAP.

\section{References}

[1] Shastri VP. Future of regenerative medicine: challenges and hurdles. Artif Organs 2006;30(10):828-34.

[2] Laschke MW, Harder Y, Amon M, Martin I, Farhadi J, Ring A, et al. Angiogenesis in tissue engineering: breathing life into constructed tissue substitutes. Tissue Eng 2006;12(8):2093-104.

[3] Zhu YB, Gao CY, He T, Shen JC. Endothelium regeneration on luminal surface of polyurethane vascular scaffold modified with diamine and covalently grafted with gelatin. Biomaterials 2004;25(3):423-30.

[4] Jain RK, Au P, Tam J, Duda DG, Fukumura D. Eng Vasc Tissue 2005;23(7):821-3.

[5] Kneser U, Polykandriotis E, Ohnolz J, Heidner K, Grabinger L, Euler S, et al Engineering of vascularized transplantable bone tissues: induction of axia vascularization in an osteoconductive matrix using an arteriovenous loop. Tissue Eng 2006;12(7):1721-31.

[6] Rouwkema J, De Boer J, Van Blitterswijk CA. Endothelial cells assemble into a 3-dimensional prevascular network in a bone tissue engineering construct. Tissue Eng 2006;12(9):2685-93.

[7] Parfitt AM. The mechanism of coupling: a role for the vasculature. Bone 2000;26(4):319-23.

[8] Brey EM, Uriel S, Greisler HP, McIntire LV. Therapeutic neovascularization: contributions from bioengineering. Tissue Eng 2005;11(3-4):567-84.

[9] Laroche M. Intraosseous circulation from physiology to disease. Joint Bone Spine 2002;69(3):262-9.

[10] Mikos AG, Herring SW, Ochareon P, Elisseeff J, Lu HH, Kandel R, et al. Engineering complex tissues. Tissue Eng 2006;12(12):3307-39.

[11] Koch S, Yao C, Grieb G, Prevel P, Noah EM, Steffens GCM. Enhancing angiogenesis in collagen matrices by covalent incorporation of VEGF. J Mater Sci Mater Med 2006;17(8):735-41.

[12] Richardson TP, Peters MC, Ennett AB, Mooney DJ. Polymeric system for dual growth factor delivery. Nat Biotechnol 2001;19(11):1029-34.

[13] Warnke PH, Springer IN, Wiltfang J, Acil Y, Eufinger H, Wehmoller M, et al. Growth and transplantation of a custom vascularised bone graft in a man. Lancet 2004;364(9436):766-70.
[14] Warnke PH, Wiltfang J, Springer I, Acil Y, Bolte H, Kosmahl M, et al. Man as living bioreactor: fate of an exogenously prepared customized tissue-engineered mandible. Biomaterials 2006;27(17):3163-7.

[15] Choong CS, Hutmacher DW, Triffitt JT. Co-culture of bone marrow fibroblasts and endothelial cells on modified polycaprolactone substrates for enhanced potentials in bone tissue engineering. Tissue Eng 2006.

[16] Levenberg S, Rouwkema J, Macdonald M, Garfein ES, Kohane DS, Darland DC, et al. Engineering vascularized skeletal muscle tissue. Nat Biotechnol 2005;23(7):879-84.

[17] Ma Z, Kotaki M, Yong T, He W, Ramakrishna S. Surface engineering of electrospun polyethylene terephthalate (PET) nano-fibers towards development of a new material for blood vessel engineering. Biomaterials 2005; 26(15):2527-36

[18] Li CM, Vepari C, Jin HJ, Kim HJ, Kaplan DL. Electrospun silk-BMP-2 scaffolds for bone tissue engineering. Biomaterials 2006;27(16):3115-24.

[19] Davis GE, Senger DR. Endothelial extracellular matrix: biosynthesis, remodeling, and functions during vascular morphogenesis and neovessel stabilization. Circ Res 2005;97(11):1093-107.

[20] Tuzlakoglu K, Bolgen N, Salgado AJ, Gomes ME, Piskin E, Reis RL. Nano- and micro-fiber combined scaffolds: a new architecture for bone tissue engineering. J Mater Sci Mater Med 2005;16(12):1099-104.

[21] Gomes ME, Sikavitsas VI, Behravesh E, Reis RL, Mikos AG. Effect of flow perfusion on the osteogenic differentiation of bone marrow stromal cells cultured on starch-based three-dimensional scaffolds. J Biomed Mater Res 2003;67A(1):87-95.

[22] Gomes ME, Azevedo HS, Moreira AR, Ella V, Kellomaki M, Reis RL. Starchpoly(epsilon-caprolactone) and starch-poly(lactic acid) fibre-mesh scaffolds for bone tissue engineering applications: structure, mechanical properties and degradation behaviour. J Tissue Eng Regen Med 2008;2(5):243-52.

[23] Jaffe EA, Nachman RL, Becker CG, Minick CR. Culture of human endothelial cells derived from umbilical veins - identification by morphologic and immunological criteria. J Clin Invest 1973;52(11):2745-56.

[24] Peters K, Schmidt H, Unger RE, Otto M, Kamp G, Kirkpatrick CJ. Softwaresupported image quantification of angiogenesis in an in vitro culture system: application to studies of biocompatibility. Biomaterials 2002;23(16):3413-9.

[25] Nachtigal P, Gojova A, Semecky V. The role of epithelial and vascular-endothelial cadherin in the differentiation and maintenance of tissue integrity. Acta Medica (Hradec Kralove) 2001;44(3):83-7.

[26] Yushkevich PA, Piven J, Hazlett HC, Smith RG, Ho S, Gee JC, et al. User-guided 3D active contour segmentation of anatomical structures: significantly improved efficiency and reliability. Neuroimage 2006;31(3):1116-28.

[27] Unger RE, Huang Q, Peters K, Protzer D, Paul D, Kirkpatrick CJ. Growth of human cells on polyethersulfone (PES) hollow fiber membranes. Biomaterials 2005;26(14):1877-84.

[28] Unger RE, Peters K, Wolf M, Motta A, Migliaresi C, Kirkpatrick CJ. Endothelialization of a non-woven silk fibroin net for use in tissue engineering: growth and gene regulation of human endothelial cells. Biomaterials 2004;25(21): 5137-46.

[29] Boura C, Muller S, Vautier D, Dumas D, Schaaf P, Claude Voegel J, et al. Endothelial cell - interactions with polyelectrolyte multilayer films. Biomaterials 2005;26(22):4568-75.

[30] Pham QP, Sharma U, Mikos AG. Electrospinning of polymeric nanofibers for tissue engineering applications: a review. Tissue Eng 2006;12(5):1197-211.

[31] Lutolf MP, Hubbell JA. Synthetic biomaterials as instructive extracellular microenvironments for morphogenesis in tissue engineering. Nat Biotechnol 2005;23(1):47-55

[32] Kwon IK, Kidoaki S, Matsuda T. Electrospun nano- to microfiber fabrics made of biodegradable copolyesters: structural characteristics, mechanical properties and cell adhesion potential. Biomaterials 2005;26(18):3929-39.

[33] Boudriot U, Dersch R, Greiner A, Wendorff JH. Electrospinning approaches toward scaffold engineering - a brief overview. Artif Organs 2006;30(10):785-92.

[34] Gomes ME, Bossano CM, Johnston CM, Reis RL, Mikos AG. In vitro localization of bone growth factors in constructs of biodegradable scaffolds seeded with marrow stromal cells and cultured in a flow perfusion bioreactor. Tissue Eng 2006;12(1):177-88.

[35] Gomes ME, Holtorf HL, Reis RL, Mikos AG. Influence of the porosity of starchbased fiber mesh scaffolds on the proliferation and osteogenic differentiation of bone marrow stromal cells cultured in a flow perfusion bioreactor. Tissue Eng 2006:12(4):801-9.

[36] Santos MI, Fuchs S, Gomes ME, Unger RE, Reis RL, Kirkpatrick CJ. Response of micro- and macrovascular endothelial cells to starch-based fiber meshes for bone tissue engineering. Biomaterials 2007;28(2):240-8.

[37] Folkman J, Shing Y. Angiogenesis. J Biol Chem 1992;267(16):10931-4.

[38] Muller AM, Hermanns MI, Cronen C, Kirkpatrick CJ. Comparative study of adhesion molecule expression in cultured human macro- and microvascular endothelial cells. Exp Mol Pathol 2002;73(3):171-80.

[39] Peters K, Unger R, Stumpf S, Schäfer J, Tsaryk R, Hoffmann B, et al. Cell typespecific aspects in biocompatibility testing: the intercellular contact in vitro as an indicator for endothelial cell compatibility. J Mater Sci Mater Med 2008;19(4):1637-44.

[40] Goldman RD, Khuon S, Chou YH, Opal P, Steinert PM. The function of intermediate filaments in cell shape and cytoskeletal integrity. J Cell Biol 1996;134(4):971-83.

[41] Cassell OC, Hofer SO, Morrison WA, Knight KR. Vascularisation of tissueengineered grafts: the regulation of angiogenesis in reconstructive surgery and in disease states. Br J Plast Surg 2002;55(8):603-10. 